\title{
Fingolimod Therapy for Multiple Sclerosis
}

\author{
Mary A. Willis, $\mathrm{MD}^{1}$ Jeffrey A. Cohen, $\mathrm{MD}^{1}$ \\ ${ }^{1}$ Neurological Institute, The Mellen Center for Multiple Sclerosis \\ Treatment and Research, Cleveland Clinic, Cleveland, Ohio \\ Semin Neurol 2013;33:37-44.
}

\begin{abstract}
Address for correspondence Jeffrey A. Cohen, MD, Neurological Institute, The Mellen Center for Multiple Sclerosis Treatment and Research, Cleveland Clinic, 9500 Euclid Avenue, Cleveland, OH 44195 (e-mail: cohenj@ccf.org).
\end{abstract}

\begin{abstract}
\section{Keywords}

- multiple sclerosis

- therapy

- fingolimod

- sphingosine 1-phosphate

Because of its potent efficacy and oral route of administration, the approval of fingolimod as treatment for relapsing-remitting multiple sclerosis (MS) was highly anticipated. The therapeutic and adverse effects are mediated by modulation of sphingosine 1-phosphate receptors. Fingolimod inhibits the egress of lymphocytes from lymph nodes and may also have direct effects on the central nervous system. The clinical trials that led to the approval of fingolimod demonstrated benefit on relapses, disability progression, magnetic resonance imaging (MRI) activity, and brain volume loss in treatment-naïve and previously treated patients with relapsing-remitting MS. The use of fingolimod in clinical practice has been limited by concerns for cardiac effects, infection, and macular edema as well as the relative lack of long-term safety data for this drug with a novel mechanism of action. Additional clinical trial and postmarketing data suggest that fingolimod is a safe, effective, and well-tolerated treatment option when patients are selected and monitored appropriately.
\end{abstract}

Multiple sclerosis (MS) is a chronic disease of the central nervous system (CNS) most often characterized by relapsing-remitting (RR) symptoms at the onset, followed by secondary progression. Inflammatory tissue damage mediated by autoreactive T cells is thought to initiate lesion formation. Variable remyelination and axonal loss result in accumulation of physical and cognitive disability. All currently approved disease-modifying therapies aim to prevent clinical relapses and limit magnetic resonance imaging (MRI) changes by modulating the inflammatory response in some way.

Until recently, all therapeutic options for RRMS required parenteral administration. Despite excellent long-term safety profiles, patient compliance with first-line drugs-interferon beta (IFN $\beta$ ) and glatiramer acetate (GA)-is limited by modest efficacy, frequent injections, and bothersome side effects. The U.S. Food and Drug Administration (FDA) approval of fingolimod (FTY720; Gilenya, Novartis, Basel, Switzerland) in September 2010, the first oral disease-modifying therapy, generated much interest among both patients and physicians.

The role of fingolimod in MS treatment continues to evolve. In this review, we describe the immunologic and CNS effects of fingolimod and the clinical trial evidence for its efficacy. We then discuss the issues to be considered in safe use of fingolimod for patients with RRMS.

\section{Mechanism of Action}

Fingolimod, a derivate of the fungal metabolite myriocin, is structurally similar to sphingosine, an important component of cell membranes. Phosphorylation by sphingosine kinase leads to formation of sphingosine 1-phosphate (S1P), which functions as a soluble regulator of a wide range of cellular processes, including lymphocyte recirculation, cardiac rate, neural cell proliferation and function, and endothelial permeability. These functions are mediated by interaction with a family of five G-protein-coupled SIP receptors. Sphingosine kinase also phosphorylates fingolimod into the active form, fingolimod-phosphate, which binds four of five S1P receptors with high affinity, acting as a functional antagonist. The therapeutic effects of fingolimod may involve S1P receptor modulation in both the immune system and the CNS. ${ }^{1-4}$

Lymphocytes, including $\mathrm{T}$ cells and B cells, predominantly express S1P-type-1 receptor $\left(\mathrm{S}_{1} \mathrm{P}_{1}\right)$, which directs lymphocyte egress from lymphoid tissues and recirculation in the periphery. Fingolimod-phosphate binds lymphocyte $\mathrm{S}_{1} \mathrm{P}_{1}$
Issue Theme Current and Emerging Therapies for Multiple Sclerosis; Guest Editor, B. Mark Keegan, MD, FRCP(C)
Copyright (c) 2013 by Thieme Medical Publishers, Inc., 333 Seventh Avenue, New York, NY 10001, USA.

Tel: +1(212) 584-4662.
DOI http://dx.doi.org/ 10.1055/s-0033-1343794. ISSN $0271-8235$. 
with high affinity resulting in receptor internalization and degradation. Levels of $\mathrm{S}_{1} \mathrm{P}_{1}$ mRNA are also reduced. ${ }^{5}$ The loss of surface expression of $\mathrm{S}_{1} \mathrm{P}_{1}$ results in functional antagonism, loss of responsiveness to the S1P gradient necessary for egress with resultant lymphocyte retention in secondary lymphoid tissue. ${ }^{1,3,5}$

The percentages of both $\mathrm{CD} 4+$ and $\mathrm{CD} 8+\mathrm{T}$ cells are reduced in fingolimod-treated patients. The predominant effect is on CC-chemokine receptor-7-positive naïve $\mathrm{T}$ cells, central memory $\mathrm{T}$ cells, and $\mathrm{T}$ cells producing interleukin17. 3,6 These subpopulations include autoreactive lymphocytes that are thought to mediate CNS inflammation in MS. Peripheral effector memory $\mathrm{T}$ cells, thought to be important in defense against local pathogen invasion, are relatively spared. $1,3,6$

Fingolimod also binds S1P receptors outside of the immune system. S1P receptors expressed by oligodendrocytes, astrocytes, microglia, and other neural cells influence neurogenesis, neural cell function, and migration. ${ }^{1,7,8}$ Fingolimod crosses the blood-brain barrier and is phosphorylated in situ. ${ }^{1,9}$ Functional antagonism of S1P receptors in the CNS may result in reduced secretion of proinflammatory cytokines, enhanced remyelination, and protection of oligodendrocytes from cell death. ${ }^{1,7,10}$ The direct effects of fingolimod in the CNS are suggested by preclinical evidence. ${ }^{10,11}$ Additional studies are needed to investigate the potential reparative and neuroprotective actions of fingolimod in MS.

\section{Pharmacology}

Once daily oral fingolimod is absorbed slowly, reaching a maximum blood concentration at 12 to 24 hours regardless of food intake. ${ }^{12,13}$ Clearance of the drug occurs predominantly by hepatic metabolism through a cytochrome $\mathrm{P} 4504 \mathrm{~F}$ pathway. ${ }^{14}$ Fingolimod is highly protein bound. The elimination half-life averages 8 to 9 days. Steady-state levels are achieved after 1 to 2 months. ${ }^{2}$ Ethnicity, gender, and mild to moderate hepatic or renal impairment do not appear to significantly affect the pharmacokinetics. ${ }^{13}$

\section{Efficacy in Clinical Trials}

A phase II proof-of-concept study randomized patients with gadolinium- (Gd-) enhancing lesions at baseline to receive oral fingolimod, $1.25 \mathrm{mg}$ or $5.0 \mathrm{mg}$, or a once-daily placebo. ${ }^{15}$ The reduction in clinical and radiographic disease in fingolimod-treated patients prompted two phase III trials. The FTY720 Research Evaluating Effects of Daily Oral Therapy in Multiple Sclerosis (FREEDOMS) study was a 24-month, double-blind, placebo-controlled study comparing two doses of fingolimod, $0.5 \mathrm{mg}$ or $1.25 \mathrm{mg}$ versus placebo. ${ }^{16}$ The same doses of fingolimod were compared with intramuscular IFN $\beta-1 a$ in the Trial Assessing Injectable Interferon Versus FTY720 Oral in Relapsing-Remitting Multiple Sclerosis (TRANSFORMS) study. ${ }^{17}$

The FREEDOMS study enrolled 1,272 patients with RRMS who had been off disease-modifying therapy for at least 3 months before randomization. The $0.5-\mathrm{mg}$ and $1.25-\mathrm{mg}$ doses of fingolimod demonstrated favorable clinical and imaging effects compared with placebo. The annualized relapse rate, the primary outcome, was lower for both doses of fingolimod (0.18-0.16) versus placebo (0.40). In addition, both doses of fingolimod significantly reduced the risk of disability progression over the 24-month study period. Patients receiving fingolimod were less likely to have new or enlarged T2-hyperintense lesions, Gd-enhancing lesions, or brain atrophy progression compared with those receiving placebo. $^{16}$

One thousand two hundred ninety-two patients were assigned to oral fingolimod, $0.5 \mathrm{mg}$ or $1.25 \mathrm{mg}$, or intramuscular IFN $\beta$ - $1 \mathrm{a}$ in the 12 -month, double-blind TRANSFORMS study. All patients had clinically active disease defined by at least one relapse during the previous year or two relapses during the previous 2 years. More than $55 \%$ of patients in all groups were previously treated with either IFN $\beta$ or GA. The proportion of patients with disability progression during the 12-month study period was small for all groups. However, the annualized relapse rate was reduced for fingolimod-treated patients (0.16-0.20) versus the active comparator group (0.33). As in the FREEDOMS study, this clinical effect was supported by superior benefit on MRI outcomes: new or enlarged T2 lesions, Gd-enhancing lesions, and brain-volume reduction in the fingolimod groups. ${ }^{17}$

Results of a phase III extension study confirmed improvements in clinical and MRI outcomes (including brain atrophy) for patients who switched from placebo to fingolimod and demonstrated sustained low clinical and MRI disease activity for patients in the continuous fingolimod-treatment group. ${ }^{18,19}$ All studies have demonstrated similar efficacy for $0.5-\mathrm{mg}$ and $1.25-\mathrm{mg}$ doses of fingolimod. Adverse effects were more common with the higher dose. Additional safety data are expected from extension studies.

\section{Safety}

The safety profile of fingolimod is based on experience with 2,615 patients in phase II and phase III studies. The risk of adverse effects (AEs) associated with a 0.5 -mg dose of fingolimod was similar to placebo in the FREEDOMS study. In both the FREEDOMS and TRANSFORMS studies, higher rates of AEs leading to drug discontinuation were associated the $1.25-\mathrm{mg}$ dose of fingolimod. The most common AEs observed with fingolimod included laboratory abnormalities, first-dose bradycardia or atrioventricular (AV) conduction slowing, macular edema, possible increased infection risk, blood pressure effects, cough, dyspnea, back pain, headache, influenza, and diarrhea. Many of the AEs can be explained by the known pharmacodynamics effects of fingolimod mediated by S1P receptors.

\section{Laboratory Abnormalities}

Lymphopenia and increased alanine aminotransferase (ALT) were the most common laboratory abnormalities. Patients receiving fingolimod demonstrated a reduction in peripheral blood lymphocyte counts within hours of the first dose. Circulating lymphocytes were reduced to 20 to $30 \%$ of 
baseline by one month. Absolute CD4 counts can be comparable to the range seen in patients with HIV infection or with myelotoxic chemotherapy. ${ }^{3}$ However, because the fingolimod-associated lymphopenia is a result of redistribution rather than depletion and effector memory $\mathrm{T}$ cells in the periphery are largely spared, these low counts do not have the same implication. Mean lymphocyte counts begin to rise within days of discontinuation and reach the lower limit of normal by 4 to 8 weeks, ${ }^{15-17}$ though prolonged lymphopenia has been reported. ${ }^{20}$ The degree of lymphopenia and its persistence after discontinuation are dose dependent.

The risk of hepatic abnormalities was also dose dependent. Increased ALT above 3 times the upper limit of normal occurred in 8 to $10 \%$ of patients who received a fingolimod $0.5-\mathrm{mg}$ dosage. ${ }^{21,22}$ Elevations in aspartate transaminase or total bilirubin were rare. No cases of symptomatic liver injury were reported in a pooled analysis of treated patients. ${ }^{21}$ Abnormalities were reversible with cessation of fingolimod. ${ }^{21,22}$

\section{Cardiac Effects}

Sphingosine 1-phosphate receptors in atrial myocytes and nodal cells are thought to play a role in heart-rate regulation. A transient decrease in heart rate and slowing of atrioventricular conduction has been consistently demonstrated after initiation of fingolimod in healthy volunteers and patients with MS. The negative chronotropic effect typically reaches a maximum at 3 to 5 hours after the first dose and can be mitigated by atropine or isoproterenol administration. Continuous Holter monitoring of healthy volunteers receiving a single daily dose of fingolimod revealed a small increase in the PR and RR intervals on day 1 with return to baseline by day 7; QT interval was not prolonged. ${ }^{23}$

Pooled analysis of data from the FREEDOMS and TRANSFORMS studies demonstrated a mean reduction of 8 beats per minute at 4 to 5 hours after receiving the first dose of $0.5-\mathrm{mg}$ fingolimod. ${ }^{21,24}$ First-degree AV block and second degree Mobitz 1 block were observed in $4.7 \%$ and $0.2 \%$ of patients receiving the FDA-approved dose. ${ }^{24}$ Most bradycardic events were asymptomatic. ${ }^{21,24}$ The effects on heart rate and AV conduction may recur upon reintroduction if fingolimod is discontinued for 2 weeks or more.

Although the transient bradycardia had limited clinical manifestations in the studies, a few postmarketing reports of serious cardiac AEs have raised concerns for patient selection and monitoring. Sustained bradycardia and brief asystole 21 hours after initiation of fingolimod was reported in a patient on long-term treatment with risperidone. ${ }^{25}$ The FDA announced investigation of a death within 24 hours after the first dose of fingolimod in a patient cotreated with metoprolol and amlodipine. ${ }^{26}$ Patients with recurrent syncope of suspected cardiac origin, known ischemic heart disease, congestive heart failure, sinus node dysfunction, resting heart rate lower than 55 beats per minute, second-degree AV block, corrected QT interval greater than $440 \mathrm{~ms}$, concomitant use of Vaughn-Williams class 3 antiarrhythmic drugs, or diabetes mellitus were excluded from the FREEDOMS and TRANSFORMS studies. Thus, experience with these higher risk patients is limited.

\section{Macular Edema}

An early study of fingolimod 2.5 and $5.0 \mathrm{mg}$ in renal transplant patients identified macular edema as a potential AE related to the medication. ${ }^{27}$ Patients in the FREEDOMS and TRANSFORMS studies were monitored with dilated funduscopic examinations and optical coherence tomography at baseline and at designated intervals throughout the study. Pooled results from phase II and phase III studies of fingolimod in MS identified 19 subjects with macular edema out of 2506 subjects treated, representing an overall incidence of 0.2 to $0.4 \%$ at the $0.5-\mathrm{mg}$ dose and 1.0 to $1.1 \%$ at the $1.25-\mathrm{mg}$ dose. $^{21,22}$ In most cases, the onset of macular edema occurred within the first 4 months of initiating therapy. Diabetes mellitus and a history of uveitis may be associated with an increased risk for macular edema. ${ }^{28}$ Fingolimod-associated macular edema usually resolves after drug discontinuation.

The North American Neuro-Ophthalmology Society and FDA recommendations include a screening evaluation for pre-existing uveitis, macular disease, or retinal vascular conditions prior to starting fingolimod. ${ }^{29,30}$ A repeat evaluation and optical coherence tomography should be done at 3 to 4 months after initiating therapy. More than $25 \%$ of the cases of macular edema associated with fingolimod have been asymptomatic. Unexplained changes in visual acuity or patient descriptions of visual distortion should prompt a referral to an ophthalmic specialist within 1 to 2 weeks.

\section{Infection}

Despite the marked reduction in peripheral lymphocyte count, the incidence of serious infections in patients treated with fingolimod was comparable to patients receiving placebo or IFN $\beta 1 \mathrm{a}$ in the phase III studies. Lower respiratory tract infections were reported slightly more often in fingolimodtreated groups (5.7-6.8\%) than in groups receiving either placebo or IFN $\beta 1 \mathrm{a}$ (3.5-4.5\%). ${ }^{22}$ The $0.5-\mathrm{mg}$ dose of fingolimod was not associated with an increased risk for herpes virus infections in the FREEDOMS or TRANSFORMS groups. One case of fatal disseminated varicella in a patient without prior exposure and one case of fatal herpes simplex encephalitis occurred in the $1.25-\mathrm{mg}$ fingolimod group in the TRANSFORMS study. Opportunistic infections such as cryptococcus, toxoplasmosis, and disseminated histoplasmosis were not seen with fingolimod treatment in clinical trials. A case of progressive multifocal leukoencephalopathy was recently reported in a John Cunningham virus- (JCV-) seropositive patient who switched from natalizumab to fingolimod. ${ }^{31}$ Due to the limited details available, the contribution from fingolimod is uncertain. The influence of prior immunosuppressant use on risk of infection with fingolimod remains unclear. The incidence of infections seen in phase III studies did not correlate with the degree of fingolimod-induced lymphopenia. ${ }^{32}$

\section{Vascular Effects}

Vascular and lymphatic endothelial cells also express $\mathrm{S}_{1} \mathrm{P}_{1}$, $\mathrm{S}_{1} \mathrm{P}_{2}$, and $\mathrm{S}_{1} \mathrm{P}_{3}$. The binding of fingolimod to these endothelial receptors produces increased permeability in some vascular beds and increased barrier function in others. ${ }^{2,33}$ The activation of $\mathrm{S} \mathrm{P}_{3}$ on vascular smooth muscle cells results in 
vasoconstriction. ${ }^{2}$ A modest, but consistent increase in blood pressure was observed in patients treated with fingolimod in the phase III trials. ${ }^{16,17}$ In addition to hypertension, the effects of fingolimod on vascular tone could also contribute to headaches. ${ }^{33}$ Rare cases of stroke, ${ }^{15}$ posterior reversible encephalopathy syndrome, ${ }^{15}$ and peripheral vasospasm ${ }^{34}$ have also been reported in patients who received higher doses of fingolimod. The small number of cases precludes confirming a causal connection to the drug. No definite procoagulant effects have been identified.

\section{Malignancy}

Although fingolimod's potent immunomodulatory effects raise concern for increased risk of malignancy, pooled data from all fingolimod trials did not demonstrate a definite association of skin carcinoma or other malignancy with fingolimod. ${ }^{22}$ Long-term follow-up is needed for adequate assessment of this risk.

\section{Pulmonary Effects}

Activation of S1P receptors expressed by airway smooth muscle cells may induce airway hyperresponsiveness. ${ }^{35,36}$ Rates of cough and dyspnea reported by fingolimod-treated patients were slightly higher than controls in phase III trials. ${ }^{16,17}$ Data from the FREEDOMS and TRANSFORMS studies demonstrated minimal decreases in forced expiratory volume at 1 second $\left(\mathrm{FEV}_{1}\right)$ and diffusing capacity for carbon monoxide $\left(D_{L} C O\right)$. $F_{1}$ effects were reversible. ${ }^{21}$ The clinical significance of these findings for patients with asthma or other pulmonary disease is uncertain. Persistent respiratory symptoms after treatment initiation should prompt pulmonary function tests, referral to a pulmonary specialist, and/or discontinuation of fingolimod.

\section{Use of Fingolimod in Clinical Practice}

Regulatory approval for once-daily dosing of 0.5-mg fingolimod as disease-modifying therapy for relapsing forms of MS was first granted in September 2010. It was approved as firstline therapy without restriction in the United States and Switzerland. Due to safety concerns described above, other agencies (Health Canada, the European Medicines Agency) approved fingolimod as second-line therapy for patients who failed IFN $\beta$ therapy or who have a rapidly progressive course. Review of cardiac concerns prompted a revision of the U.S. prescribing information in April 2012. ${ }^{29}$

Fingolimod should be used as monotherapy. The safety and efficacy of fingolimod combined with other disease-modifying agents has not been established. Fingolimod does not interact significantly with other drugs used to manage MS symptoms, including fluoxetine, paroxetine, carbamazepine, baclofen, gabapentin, oxybutynin, amantadine, modafinil, amitriptyline, pregabalin, and corticosteroids.

Fingolimod is not approved for use in children or adolescents. Experience in elderly patients is limited. Fingolimod was observed to be teratogenic in rats. ${ }^{27}$ As a result, although some pregnancies during fingolimod therapy have resulted in healthy delivery, women of child-bearing age should use effective contraception during fingolimod treatment and for 2 months after stopping therapy. ${ }^{37}$

Although prediction of the potential AEs discussed above is challenging, pretreatment screening tests advised by regulatory agencies helps ensure appropriate patient selection. Our recommendations for pretreatment screening and follow-up monitoring are summarized in -Table 1. Recently updated FDA recommendations for first-dose monitoring are listed in - Table 2. Blood pressure and heart rate should be monitored hourly for 6 hours after the initial dose, and an electrocardiogram should be performed predosing and before discharge at 6 hours. Patients with a low resting heart rate or those taking certain medications ( $\beta$ blockers, calcium channel blockers, or psychotropic agents) may require prior cardiology evaluation or overnight monitoring following first-dose administration.

Although some patients in the fingolimod trials had highly active disease prior to enrollment, this subgroup has not been specifically studied. A patient with two severe relapses, 3point Extended Disability Status Scale worsening, and an increase in MRI activity within the first 3 months of fingolimod treatment has been described. ${ }^{38}$ A patient who developed encephalopathy and bilateral, extensive brain lesions during fingolimod treatment was later found to have antiaquaporin-4 antibodies. ${ }^{39}$ Alternative diagnoses, such as neuromyelitis optica, should be excluded for patients who experience dramatic worsening on fingolimod treatment. Possible rebound in disease activity after fingolimod discontinuation has been described. ${ }^{40}$

\section{Place in Treatment Algorithm}

Despite its approval as a first-line agent in the United States, relatively limited clinical experience and concerns for rare, but potentially significant AEs has limited the use of fingolimod as initial therapy. The long-term safety profiles of IFN $\beta$ and GA are well established and recommended monitoring is less stringent. Thus, these medications remain the preferred first-line agents at present. Fingolimod is a reasonable alternative for patients with RRMS who have intolerable side effects, poor compliance, or inadequate therapeutic response. Natalizumab would also be an appropriate option for such patients. Factors influencing risk-benefit ratio in individual patients (such as JCV seropositivity) should be considered. - Fig. $\mathbf{1}$ is an algorithm reflecting our current practice. Like Pelletier and Hafler, ${ }^{41}$ we tend to favor fingolimod over natalizumab in JCVseropositive patients, particularly with prior immunosuppressant therapy. The utility of fingolimod versus natalizumab in JCV-seronegative patients remains to be determined.

Patients treated with IFN $\beta$ or GA may be switched directly to fingolimod. Generally, natalizumab-treated patients should remain off therapy for 3 months prior to initiation of fingolimod. The washout should be reduced to 1 to 2 months in highly active patients. Patients who discontinue fingolimod therapy should not receive immunosuppressive medications until lymphocyte counts are within the normal range (typically within 2 months of drug cessation). Experience in the use of fingolimod after monoclonal antibodies 
Table 1 Suggested fingolimod monitoring

\begin{tabular}{|l|}
\hline Pretreatment \\
\hline Complete blood count, liver function tests \\
\hline $\begin{array}{l}\text { Document history of chicken pox or test for varicella zoster antibodies } \\
\text { If no immunity to varicella zoster, administer the two-stage chicken pox vaccine and delay therapy for } 1 \text { mo. }\end{array}$ \\
\hline $\begin{array}{l}\text { Electrocardiogram } \\
\text { If abnormal, refer to cardiology before initiating therapy. }\end{array}$ \\
\hline Ophthalmologic exam and/or optical coherence tomography \\
\hline $\begin{array}{l}\text { Pulmonary function tests and/or pulmonary referral in patients with asthma, chronic obstructive pulmonary disease, smoking } \\
\text { history, or other pulmonary condition }\end{array}$ \\
\hline Pregnancy test for women with reproductive potential \\
\hline MRI brain with and without contrast to establish new baseline prior to starting treatment ${ }^{\text {a }}$ \\
\hline Ongoing monitoring \\
\hline $\begin{array}{l}\text { Ophthalmologic exam and/or optical coherence tomography at 3-4 mo after treatment initiation and for reports of vision } \\
\text { disturbances }\end{array}$ \\
\hline Complete blood count, liver function tests at 3 and 6 mo after treatment initiation ${ }^{\text {a }}$ \\
\hline MRI brain after 1 y on treatment or earlier for unexpected manifestations ${ }^{\text {a }}$ \\
\hline Pulmonary referral and/or pulmonary function tests for respiratory symptoms \\
\hline Monitor for signs/symptoms of infection; consider suspending treatment for serious infection \\
\hline
\end{tabular}

Abbreviations: MRI, magnetic resonance imaging.

author opinion; not a U.S. Food and Drug Administration recommendation.

Table 2 Revised U.S. prescribing information for fingolimod

Contraindications

Recent (within 6 mo) myocardial infarction, unstable angina, stroke, transient ischemic attack, decompensated congestive heart failure requiring hospitalization or class III/IV congestive heart failure

History or presence of Mobitz type II second- or third-degree atrioventricular block or sick sinus syndrome, unless the patient has a pacemaker

Baseline QTc interval > $500 \mathrm{~ms}$

Treatment with class la (quinidine, procainamide) and class III (amiodarone) antiarrhythmic drugs

First-dose monitoring

Observe for signs/symptoms of bradycardia for at least $6 \mathrm{~h}$ after first dose

Hourly heart rate and blood pressure

Electrocardiogram prior to dosing and after 6-h observation

Extend monitoring if:

Lowest heart rate occurs at $6 \mathrm{~h}$ after dose

Heart rate $<45$ bpm, new onset second-degree atrioventricular block or higher, symptomatic bradycardia

Indications for overnight monitoring with continuous electrocardiogram

Pharmacological intervention needed to treat bradycardia

Prolonged QTc interval (> 450 millisecondsec males, > 470 millisecondsec females) at baseline or during observation

Additional risk for QT prolongation (e.g., hypokalemia, hypomagnesemia, congenital long-QT, concomitant QT-prolonging drugs)

Patients expected to be less tolerant of fingolimod-induced bradycardia or at increased risk because of pre-existing medical condition or concomitant heart-rate-lowering drug

Reinitiation of therapy requires first-dose precautions

Interrupted $>1$ day during weeks $1-2$ of therapy

Interrupted $>7$ days during weeks 3-4 of therapy

Interrupted $>14$ days after 1 mo of therapy 


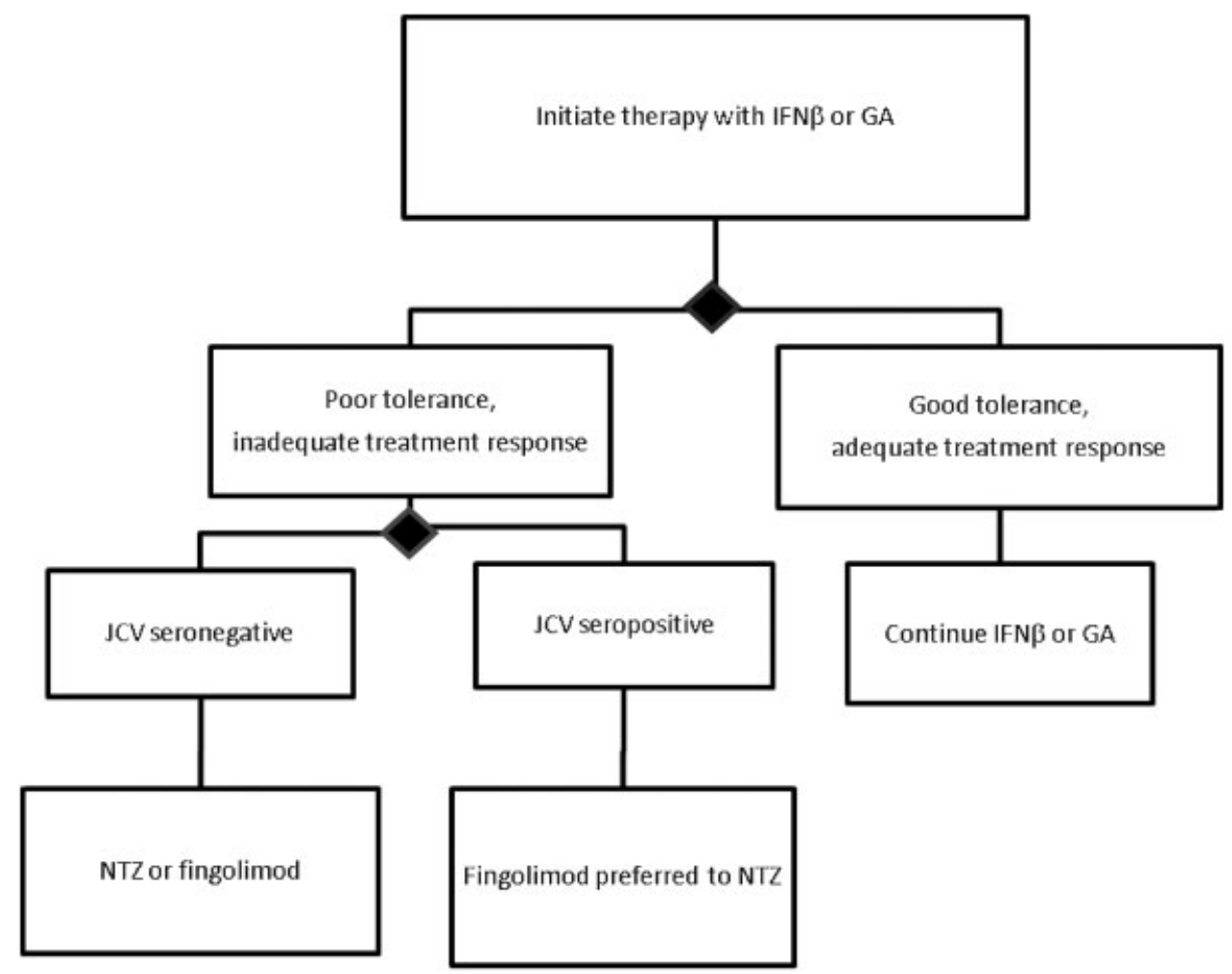

Fig. 1 Proposed treatment algorithm for relapsing remitting multiple sclerosis. IFN $\beta$, interferon beta; GA, glatiramer acetate; JCV, John Cunningham virus; NTZ, natalizumab.

that produce prolonged lymphocyte depletion such as alemtuzumab or rituximab is limited.

\section{Future Directions}

Future studies and clinical experience will better define the role of fingolimod in MS treatment. Long-term safety data will address concerns for risk of opportunistic infections, neoplasms, and reproductive effects. An ongoing 3-year, phase III trial in primary progressive MS will evaluate the potential neuroprotective and/or reparative actions of fingolimod. ${ }^{42}$ Additional studies will help establish the safety and efficacy of fingolimod in RRMS patients with inadequate treatment response to other currently approved medications. ${ }^{43}$ Studies of lower dose fingolimod and agents that bind S1P receptors more selectively may suggest mechanisms to improve the risk-to-benefit ratio of oral S1P modulators. ${ }^{44-46}$

\section{Conclusion}

The availability of oral fingolimod provides an important new option for management of MS. Modulation of S1P receptors on lymphocytes limits circulation of immune cells thought to be responsible for initiation of inflammatory damage. Fingolimod may also have direct beneficial effects on the CNS. Although the efficacy, tolerability, and safety have been demonstrated in large clinical trials, uncertainty surrounding cardiac AEs and long-term safety probably will reduce its use as first-line therapy for the near future. Ongoing studies will further define the role of fingolimod in the changing landscape of MS care.

\section{Acknowledgments}

Dr. Willis is supported by a Biogen Idec Clinical Fellowship Award. Dr. Cohen has received personal compensation for consulting or speaking from Biogen Idec, Novartis, Teva, and Vaccinex; and research support from Biogen Idec, Novartis, Genzyme, Teva, Receptos, Synthon, and Vaccinex.

\section{References}

1 Chun J, Hartung HP. Mechanism of action of oral fingolimod (FTY720) in multiple sclerosis. Clin Neuropharmacol 2010;33 (2):91-101

2 Cohen JA, Chun J. Mechanisms of fingolimod's efficacy and adverse effects in multiple sclerosis. Ann Neurol 2011;69(5):759-777

3 Mehling M, Johnson TA, Antel J, Kappos L, Bar-Or A. Clinical immunology of the sphingosine 1-phosphate receptor modulator fingolimod (FTY720) in multiple sclerosis. Neurology 2011; 76(8, Suppl 3):S20-S27

4 Mehling MKL, Kappos L, Derfuss T. Fingolimod for multiple sclerosis: mechanism of action, clinical outcomes, and future directions. Curr Neurol Neurosci Rep 2011;11(5):492-497

5 Gräler MHGE, Goetzl EJ. The immunosuppressant FTY720 downregulates sphingosine 1 -phosphate $\mathrm{G}$-protein-coupled receptors. FASEB J 2004;18(3):551-553

6 Mehling MBV, Brinkmann V, Antel J, et al. FTY720 therapy exerts differential effects on $T$ cell subsets in multiple sclerosis. Neurology 2008;71(16):1261-1267 
7 Brinkmann V, Billich A, Baumruker T, et al. Fingolimod (FTY720): discovery and development of an oral drug to treat multiple sclerosis. Nat Rev Drug Discov 2010;9(11):883-897

8 Dev KK, Mullershausen F, Mattes H, et al. Brain sphingosine-1phosphate receptors: implication for FTY720 in the treatment of multiple sclerosis. Pharmacol Ther 2008;117(1):77-93

9 Foster CA, Howard LM, Schweitzer A, et al. Brain penetration of the oral immunomodulatory drug FTY720 and its phosphorylation in the central nervous system during experimental autoimmune encephalomyelitis: consequences for mode of action in multiple sclerosis. J Pharmacol Exp Ther 2007;323(2):469-475

10 Miron VE, Schubart A, Antel JP. Central nervous system-directed effects of FTY720 (fingolimod). J Neurol Sci 2008;274(1-2):13-17

11 Coelho RP, Payne SG, Bittman R, Spiegel S, Sato-Bigbee C. The immunomodulator FTY720 has a direct cytoprotective effect in oligodendrocyte progenitors. J Pharmacol Exp Ther 2007;323 (2):626-635

12 Kovarik JM, Hartmann S, Bartlett M, et al. Oral-intravenous crossover study of fingolimod pharmacokinetics, lymphocyte responses and cardiac effects. Biopharm Drug Dispos 2007;28 (2):97-104

13 Kovarik JM, Schmouder R, Barilla D, Wang Y, Kraus G. Single-dose FTY720 pharmacokinetics, food effect, and pharmacological responses in healthy subjects. Br J Clin Pharmacol 2004;57(5):586591

14 Warnke C, Stüve O, Hartung HP, Fogdell-Hahn A, Kieseier BC. Critical appraisal of the role of fingolimod in the treatment of multiple sclerosis. Neuropsychiatr Dis Treat 2011;7:519-527

15 Kappos L, Antel J, Comi G, et al; FTY720 D2201 Study Group. Oral fingolimod (FTY720) for relapsing multiple sclerosis. N Engl J Med 2006;355(11):1124-1140

16 Kappos L, Radue EW, O'Connor P, et al; FREEDOMS Study Group. A placebo-controlled trial of oral fingolimod in relapsing multiple sclerosis. N Engl J Med 2010;362(5):387-401

17 Cohen JA, Barkhof F, Comi G, et al; TRANSFORMS Study Group. Oral fingolimod or intramuscular interferon for relapsing multiple sclerosis. N Engl J Med 2010;362(5):402-415

18 Kappos L, Radue E-W, O' Connor P, et al. Long-term efficacy and safety of fingolimod (FTY720) in relapsing-remitting multiple sclerosis (RRMS): results from the extension of the phase III FREEDOMS study. Paper presented at: Annual Meeting of the American Academy of Neurology; April 26, 2012; New Orleans, LA

19 Antel J, Montalban X, O'Connor P, et al. Long-term (7-year) data from a phase 2 extension study of fingolimod in relapsing multiple sclerosis. Paper presented at: Annual Meeting of the American Academy of Neurology; April 23, 2012; New Orleans, LA

20 Johnson TA, Shames I, Keezer M, et al. Reconstitution of circulating lymphocyte counts in FTY720-treated MS patients. Clin Immunol 2010;137(1):15-20

21 U.S. Food and Drug Administration. (FDA) Center for Drug Evaluation and Research (CDER). Fingolimod Background Package (NDA 22-257). Available at: http://www.fda.gov/downloads/advisorycommittees/committeesmeetingmaterials/drugs/peripheralandcentralnervoussystemdrugsadvisorycommittee/ucm216553.pdf. Accessed May 6, 2012

22 Collins W, Cohen J, O' Connor P, et al. Long-term safety of oral fingolimod (FTY720) in relapsing multiple sclerosis: integrated analyses of phase 1 and phase 3 studies (P843). Mult Scler 2010;16 (Suppl 10):S295

23 Schmouder R, Serra D, Wang Y, et al. FTY720: placebo-controlled study of the effect on cardiac rate and rhythm in healthy subjects. J Clin Pharmacol 2006;46(8):895-904

24 DiMarco JP, O'Connor P, Cohen JA, et al. First-dose effect of fingolimod: pooled safety data from two phase 3 studies (TRANSFORMS and FREEDOMS). Mult Scler 2010;16(Suppl 10):S290

25 Espinosa PS, Berger JR. Delayed fingolimod-associated asystole. Mult Scler 2011;17(11):1387-1389
26 U.S. Food and Drug Administration. FDA Drug Safety Communication: Safety Review of a Reported Death after the First Dose of Multiple Sclerosis Drug Gilenya (Fingolimod). Available at: http:// www.fda.gov/Drugs/DrugSafety/ucm284240.htm. Accessed May 6, 2012

27 Salvadori M, Budde K, Charpentier B, et al; FTY720 0124 Study Group. FTY720 versus MMF with cyclosporine in de novo renal transplantation: a 1-year, randomized controlled trial in Europe and Australasia. Am J Transplant 2006;6(12):2912-2921

28 Jain N, Bhatti MT. Fingolimod-associated macular edema: incidence, detection, and management. Neurology 2012;78(9):672680

29 U.S. Food and Drug Administration. FDA Approved Labeling Text for GILENYA (Fingolimod) Capsules (NDA 02257). Updated April 2012. Washington, DC: U.S. Food and Drug Administration

30 ONE Neuro-Ophthalmology Committee. Oral MS Drug May Increase Risk for Macular Edema: A Report from the North American Neuro-Ophthalmology Society (NANOS) and the AAO. Released September 10, 2011. Available at: http://www.nanosweb.org/ files/public/MS_drug_may_increase_risk_for_RVO1NOTRACK2lf. pdf. Accessed May 6, 2012

31 Novartis. Gilenya (Finglimod) Safety Information Update (Released April 13, 2012). Available at: http://www.novartis.com/ downloads/newsroom/product-related-info-center/statementPML.pdf. Accessed May 15, 2012

32 Francis GS, Kappos L, O' Connor P, et al. Lymphocytes and fingolimod-temporal pattern and relationship with infections. Mult Scler 2010;16(Suppl 10):S146

33 Ontaneda D, Cohen JA. Potential mechanisms of efficacy and adverse effects in the use of fingolimod (FTY720). Expert Rev Clin Pharmacol 2011;4(5):567-570

34 Schwarz A, Korporal M, Hosch W, Max R, Wildemann B. Critical vasospasm during fingolimod (FTY720) treatment in a patient with multiple sclerosis. Neurology 2010;74(24):2022-2024

35 Pfaff M, Powaga N, Akinci S, et al. Activation of the SPHK/S1P signalling pathway is coupled to muscarinic receptor-dependent regulation of peripheral airways. Respir Res 2005;6:48

36 Brinkmann V, Baumruker T. Pulmonary and vascular pharmacology of sphingosine 1-phosphate. Curr Opin Pharmacol 2006;6 (3):244-250

37 Collins W, Francis G, Koren G, et al. Lack of interaction between fingolimod (FTY720) and oral contraceptives, and pregnancy experience in the clinical program of fingolimod in multiple sclerosis. Paper presented at: Annual Meeting of the American Academy of Neurology; April 9, 2011; Honolulu, HI

38 Castrop F, Kowarik MC, Albrecht H, et al. Severe multiple sclerosis relapse under fingolimod therapy: incident or coincidence? Neurology 2012;78(12):928-930

39 Min J-H, Kim BJ, Lee KH. Development of extensive brain lesions following fingolimod (FTY720) treatment in a patient with neuromyelitis optica spectrum disorder. Mult Scler 2012;18(1): 113-115

40 Havla JB, Pellkofer HL, Meinl I, Gerdes LA, Hohlfeld R, Kümpfel T. Rebound of disease activity after withdrawal of fingolimod (FTY720) treatment. Arch Neurol 2012;69(2):262-264

41 Pelletier D, Hafler DA. Fingolimod for multiple sclerosis. N Engl J Med 2012;366(4):339-347

42 Novartis. FTY720 in Patients With Primary Progressive Multiple Sclerosis. ClinicalTrials.gov Identifier: NCT00731692. Available at: http://ClinicalTrials.gov/show/NCT00731692. Accessed May 15, 2012

43 Novartis. Disease Control and Safety in Patients With Relapsing Remitting Multiple Sclerosis (RRMS) Switching From Natalizumab to Fingolimod. ClinicalTrials.gov Identifier: NCT01499667. Available at: http://ClinicalTrials.gov/show/NCT01499667. Accessed May 15, 2012

44 GlaxoSmithKline. A Study to Assess the Relative Bioavailability of Different Formulations of GSK2018682, a Sphingosine- 
44 Fingolimod Therapy for Multiple Sclerosis Willis, Cohen

1-Phosphate Receptor Subtype 1 Agonist, in Healthy Volunteers. ClinicalTrials.gov Identifier: NCT01466322. Available at: http:// ClinicalTrials.gov/show/NCT01466322. Accessed May 15, 2012

45 Novartis. Safety, Tolerability, Efficacy and Optimal Dose Finding Study of BAF312 in Patients With Relapsing-Remitting Multiple Sclerosis. ClinicalTrials.gov Identifier: NCT00879658. Available at:
http://ClinicalTrials.gov/show/NCT00879658. Accessed May 15, 2012

46 Ono Pharma A Study of the Safety and Efficacy of ONO-4641 in Patients With Relapsing-Remitting Multiple Sclerosis. ClinicalTrials.gov Identifier: NCT01081782. Available at: http://ClinicalTrials.gov/show/NCT01081782. Accessed May 15, 2012 